\title{
Effects of Abrupt Variations of Solar Wind Dynamic Pressure on the High-Latitude Ionosphere
}

\author{
Igino Coco, ${ }^{1}$ Ermanno Amata, ${ }^{1}$ Maria Federica Marcucci, ${ }^{1}$ \\ Danila Ambrosino, ${ }^{1}$ and Simon G. Shepherd ${ }^{2}$ \\ ${ }^{1}$ Istituto di Fisica dello Spazio Interplanetario (IFSI), Istituto Nazionale di Astrofisica (INAF), \\ Via del Fosso del Cavaliere, 100, 00133 Rome, Italy \\ ${ }^{2}$ Thayer School of Engineering at Dartmouth, Dartmouth College, 8000 Cummings Hall, Hanover, NH 03755-8000, USA
}

Correspondence should be addressed to Igino Coco, igino.coco@ifsi-roma.inaf.it

Received 16 February 2011; Accepted 8 April 2011

Academic Editor: Lucilla Alfonsi

Copyright () 2011 Igino Coco et al. This is an open access article distributed under the Creative Commons Attribution License, which permits unrestricted use, distribution, and reproduction in any medium, provided the original work is properly cited.

\begin{abstract}
We show the results of a statistical study on the effects in the high-latitude ionosphere of abrupt variations of solar wind dynamic pressure, using Super Dual Auroral Radar Network (SuperDARN) data in both hemispheres. We find that, during periods of quiet ionospheric conditions, the amount of radar backscatter increases when a variation in the dynamic pressure occurs, both positive (increase of the pressure) and negative (decrease of the pressure). We also investigate the behaviour of the Cross-Polar Cap Potential (CPCP) during pressure variations and show preliminary results.
\end{abstract}

\section{Introduction}

The variations of solar wind dynamic pressure are known to affect the energy and momentum transfer from the solar wind to the magnetosphere-ionosphere system. To this respect, two important factors are the rise time and the duration of the pressure perturbation. For short rise times strong transient perturbations are observed both in the magnetosphere and in the ionosphere until a new location of the magnetopause boundary is established (e.g., $[1,2])$. On the other hand, the duration of a pressure perturbation determines whether the effects will be localized or global [3]. If the duration is long enough to engulf most of the magnetosphere in the solar wind region of enhanced/reduced pressure, the pressure variation causes typical global increases of the geomagnetic field strength measured at the geostationary orbit, and on the ground at equatorial and middle latitudes, which are usually called Sudden Commencements (SC) or Sudden Impulses (SI), depending on whether they are followed by a geomagnetic storm (SC), or not (SI); in such cases, often one refers to the pressure variation itself as an SI, and this is the notation we will follow from now on.
The energy transfer from the solar wind to the magnetosphere and the ionosphere during SIs can be huge, especially when the Interplanetary Magnetic Field (IMF) has a negative $B_{z}$ component. This has been shown for example by Boudouridis et al. [4], who calculated the geoeffectiveness of a number of pressure variations as the ratio between the variation of the Cross-Polar Cap Potential (CPCP), that is, the maximum electrostatic potential difference in the polar cap, as measured by DMSP ionospheric satellites, and the potential driven by the solar wind across the magnetosphere, obtained as the product of the solar wind electric field and the variation of the magnetospheric size due to the pressure pulse. The CPCP is often considered as a good proxy for the energy transfer between the solar wind and the magnetosphere-ionosphere system [5]. They found that the geoeffectiveness, or the solar wind-magnetosphereionosphere coupling efficiency can increase by about $80 \%$ during a pressure variation with negative IMF $B_{z}$. Moreover, some case studies have shown that even when IMF $B_{z}$ is positive, the occurrence of an abrupt pressure variation can favor reconnection processes in the polar cusps and stimulate energy injection in the polar cap $[6,7]$. The present work is aimed at describing, from a statistical point of 
view, the effects of SIs on the high-latitude ionosphere by means of Super Dual Auroral Radar Network (SuperDARN) measurements $[8,9]$. Here we recall that the SuperDARN radars are based on coherent backscatter: the emitted radio signals are backscattered by density irregularities in the ionosphere, which follow the motion of the ambient plasma, so that the autocorrelation functions of the lag times of the backscattered power allow reconstruction of the Doppler velocities of the plasma structures in the ionosphere with respect to the ground. The SuperDARN radars monitor the high-latitude ionosphere in both Hemispheres simultaneously and continuously, detecting the backscattered signals along 16 directions (beams) and 75 range gates along each beam, for a total field of view of about $52^{\circ}$ for each radar.

Auroral oval expansions to lower latitudes have been often observed after pressure variations (e.g., Zhou and Tsurutani [10]): this means the particle precipitation increases and modifies the ionospheric density. Precipitation affects the plasma gradients in the ionosphere at every spatial scale from hundreds of kilometers to meters, thus affecting the radar backscatter as well (e.g., Villain et al. [11]). The effects of geomagnetic disturbances induced by interplanetary drivers on the radar echoes have been recently investigated (e.g., Ballatore et al. [12]; Wild and Grocott [13]). A good correlation between the echo activity and the IMF $B_{z}$ component and the solar wind electric field (or the reconnection rate) has been shown; furthermore, the global level of scatter seems to maximize prior to the substorm onsets and, in the nightside ionosphere, backscatter poleward of $\sim 70^{\circ}$ magnetic latitude is reduced, with radar echoes shifting to lower latitudes.

A previous work by Coco et al. [14] (Co2005 in the following) reported clear effects on the Northern Hemisphere (NH) SuperDARN echoes following the SIs taking into account 178 events from November 1997 until September 2000; in more detail, the authors found an increase of the echoes closely related to solar wind dynamic pressure enhancements (positive SIs), during periods with low ionospheric activity (as monitored by the AE index level). The database of the SIs has now been extended until the end of year 2004, including Southern Hemisphere $(\mathrm{SH})$ radar data. In Section 2 we will briefly recall the analysis technique already used by Co2005, with some new features, and in Section 2.1 we will describe the results for the new extended database in the Northern and Southern hemispheres. A first attempt to evaluate the CPCP in a statistical way for our new set of events has been outlined in Section 3, as regards both the analysis technique and the description of preliminary results. Discussion and conclusions are presented in Section 4.

\section{Data Base and Analysis Method}

We selected about 300 events of pressure variations from ACE data (October 2000-December 2004) with the following characteristics:

(1) solar wind dynamic pressure roughly constant over at least 30 minutes before and after the SI $(Q \leq 0.5$ where $Q=2 \sigma_{p} /|p|,|p|$ is the average of the dynamic pressure over the 30 -minute periods and $\sigma_{p}$ is the standard deviation),

(2) $|\Delta p|>3 \mathrm{nPa}$, where $\Delta p$ is the jump (positive or negative) in pressure,

(3) $\Delta t \leq 10 \mathrm{~min}$, where $\Delta t$ is the rise (fall) time of the pressure jump.

The selection criteria aim to isolate clear pressure variations whose duration is long enough to cause a global effect on the magnetosphere-ionosphere system: a pressure discontinuity which travels on average with a speed of $300 \div 400 \mathrm{~km} / \mathrm{s}$, covers between 80 and $120 R_{E}$ in 30 minutes (point 1), thus involving the whole magnetospheric cavity in the interaction; the other criteria (points 2 and 3) are arbitrarily chosen after careful inspection of a preliminary larger data base of pressure variations and represent typical limits for shocks.

We then divided the events in two classes, Increases (Is) and Decreases (Ds) of solar wind dynamic pressure, and we took and inspected all available data of IMF and solar wind parameters in a 2-hour period centred at the event. In the previous study, for each event Co2005 calculated a delay to account for propagation from WIND position to the centre of the Earth, simply as

$$
\Delta \tau(\mathrm{ev})=\frac{X_{\mathrm{WIND}}}{V_{\mathrm{SW}}(X)},
$$

where $X_{\text {WIN/ACE }}$ is the position of the spacecraft along the GSM $X$ direction, and $V_{\mathrm{SW}}(X)$ is the component of the solar wind velocity along the same direction. On this basis they defined an expected event time on the ground as $T_{g}=T_{\mathrm{WIND}}+\Delta \tau$. One has to bear in mind, however, that the delay calculated as in (1) is not very accurate, due to possible propagation effects of the pressure discontinuities from L1 to Earth. For that reason a better evaluation of the delay time has been performed, looking at the response of 5 ground-based magnetometers close to the geomagnetic equator. 1-minute resolution data from these stations have been visually inspected and, when a clear SI effect (sudden increase/decrease of the $H$ component) was detected, the time when the effect began was taken as $T_{g}$. Otherwise, $T_{g}$ has been calculated as in (1), using ACE data instead of WIND.

The effects of a SI as observed at ground may result from the superposition of effects depending on various conditions in the ionosphere, in the magnetosphere, and in the solar wind; therefore, it is reasonable to group the events into subsets, to study the echo response for each of them. The most relevant parameter to group the events is the Auroral Electrojet index (AE), introduced by Davis and Sugiura [15] to monitor the occurrence of auroral phenomena and more generally magnetospheric substorms. For each event we built a data base comprising of (1) one hour of AE index (with 1minute resolution) data centered on $T_{g}$, and (2) one hour of SuperDARN data (with 2-minutes resolution), also centred on $T_{g}$, from all the available radars in the $\mathrm{NH}$ and $\mathrm{SH}$.

Let $M=\langle\mathrm{AE}\rangle+\sigma$, and $Q=(2 \sigma) /\langle\mathrm{AE}\rangle$, where $\langle\mathrm{AE}\rangle$ is the time average of $\mathrm{AE}$ and $\sigma$ its standard deviation 
calculated over a $[-30,10]$ min interval about $T_{g}$. In this regard, a natural choice would have been the $[-30,0] \mathrm{min}$ interval; however, we extended it by $10 \mathrm{~min}$ to allow for the remaining uncertainties in the $T_{g}$ determination. We define the following:

(i) Quiet event: an event which satisfies one of the following conditions:

(1) $M<200 \mathrm{nT}$,

(2) $200 \leq M \leq 300 \mathrm{nT}$, and $Q \leq 0.5$.

(ii) Disturbed event: an event which satisfies one of the following conditions:

(1) $M>400 \mathrm{nT}$,

(2) $300 \leq M \leq 400 \mathrm{nT}$, and $Q \geq 0.5$.

(iii) Intermediate event: an event which satisfies one of the following conditions:

(1) $200 \leq M \leq 300 \mathrm{nT}$, and $Q>0.5$,

(2) $300 \leq M \leq 400 \mathrm{nT}$, and $Q<0.5$.

The Intermediate class has the purpose of clearly separating the Quiet and Disturbed events. Consequently, Intermediate events, 42 in number ( 24 Is and 18 Ds), are excluded from the following analysis.

In Co2005 the details of the analysis are described. For each event of the data base a Rate of Scattering (RS) is calculated as a function of time, as the percent ratio of the number of detected echoes during a 2-minute time bin, and the total number of echoes over the one-hour interval of the event. We excluded the echoes coming from ground scatter and we retained only the ionospheric echoes for which the signal-to-noise ratio was greater than $6 \mathrm{~dB}$, and the events for which the total number of echoes was greater than 5000 . Then, a Mean Rate of Scattering (MRS) as a function of time is built for each subset of events, simply as the average over the number of events in the set, of the RS of the single events (equation $3 \div 5$ in Section 3 of Co2005). Through the standard deviation $\sigma\left(t_{i}\right)$ of the MRS, where $t_{i}$ is a 2-minute time bin, a statistical error $\epsilon\left(t_{i}\right)=\sigma\left(t_{i}\right) / \sqrt{N}$, where $N$ is the number of events of the set, is associated to the MRS of each bin.

2.1. Results for the Echoes Statistics. In Table 1 information about the statistics are summarized. SuperDARN coverage is less abundant in the $\mathrm{SH}$ with respect to the $\mathrm{NH}$, and this is evident in the total number of events and echoes, practically halved in the SH for all the subsets. Moreover, one can note the number of Ds is almost half the number of Is; this stems from the nature itself of the pressure decrease phenomena: very often pressure perturbations are driven by shock fronts in the solar wind which compress the plasma ahead of them, leaving broad regions of denser plasma behind, whose contours are not so neat and sharp both in space and time. The consequence of this is that steep and strong pressure decreases are less common than increases. The definition
TABLE 1: An overview of the echoes statistics for the different subsets of the events under study. Is and Ds stand for Increases and Decreases of solar wind dynamic pressure, $\mathrm{NH}$ and $\mathrm{SH}$ stand for Northern and Southern Hemisphere.

\begin{tabular}{lcc}
\hline Set of events & Number of events & Number of echoes \\
\hline Quiet Is NH & 73 & 2827600 \\
Quiet Is SH & 50 & 907922 \\
Quiet Ds NH & 35 & 1550229 \\
Quiet Ds SH & 29 & 621471 \\
Disturbed Is NH & 73 & 2490800 \\
Disturbed Is SH & 54 & 1091885 \\
Disturbed Ds NH & 42 & 1823510 \\
Disturbed Ds SH & 35 & 652196 \\
\hline
\end{tabular}

of the RS and MRS are therefore appropriate in order to make the statistical results show up, regardless of the different numbers of echoes and events in the different subsets. Of course a risk exists that events with very few echoes influence too much the statistics: for that reason, a threshold of 5000 echoes has been set in order to accept or discard an event, which seems reasonable considering the average number of echoes per event is about 40000 for $\mathrm{NH}$ and 20000 for SH.

Figure 1 shows the MRS as a function of the scan time, $t_{i}$, for Quiet events. $T=0$ corresponds to $T_{g}$. Figures 1(a) and 1 (c) refer to the $\mathrm{NH}$, while Figures $1(\mathrm{~b})$ and $1(\mathrm{~d})$ refer to the SH; Figures 1(a) and 1(b) refer to the Is, Figures 1(c) and $1(d)$ to the Ds. The MRS trends show a clear rise of the echoes in both Hemispheres, and for both Is and Ds starting close to $T_{g}$, more evident for the $\mathrm{SH}$.

Figure 2 shows the same quantity for Disturbed events, with the same arrangement as for Figure 1. The set of Disturbed events is far less homogeneous than the Quiet events set: this stems from its definition and results from a careful inspection of the single events, as well. Therefore, the MRS trends are more difficult to interpret and understand. In the $\mathrm{NH}$ a rising trend of the MRS is observed for both Is and Ds, starting 4-5 minutes after $T_{g}$. In the $\mathrm{SH}$ no clear trends can be inferred from data.

\section{The Cross-Polar Cap Potential}

Maps of the high-latitude CPCP have been calculated for each event using the technique of Ruohoniemi and Baker [16]: the polar cap potential contours are obtained through a spherical harmonic expansion, whose coefficients are determined through a least square fit of the measured SuperDARN line-of-sight velocities; where data coverage is poor ar absent, data from the empirical model of Ruohoniemi and Greenwald [17] (RG96) have been put in the maps. The RG96 model consists in a superposed epoch analysis of many years of SuperDARN convection velocity measurements and gives average pictures of the ionospheric convection as a function of orientation and strength of the IMF. The use of the model data in the fit is extensively explained in Shepherd and Ruohoniemi [18]: the number of model vectors added to 


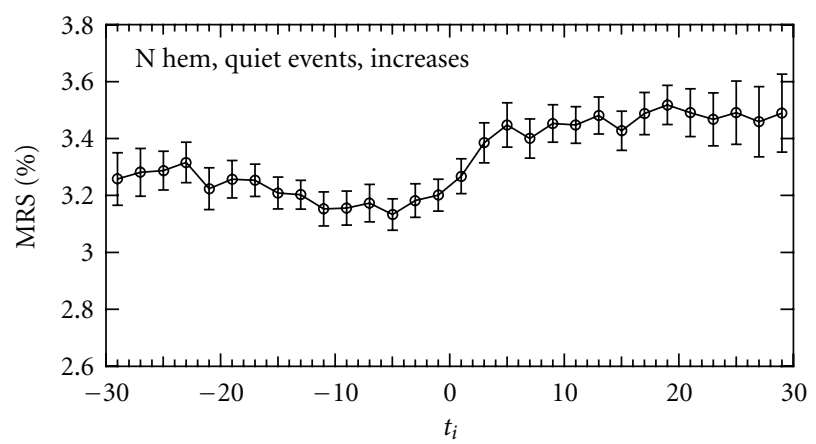

(a)

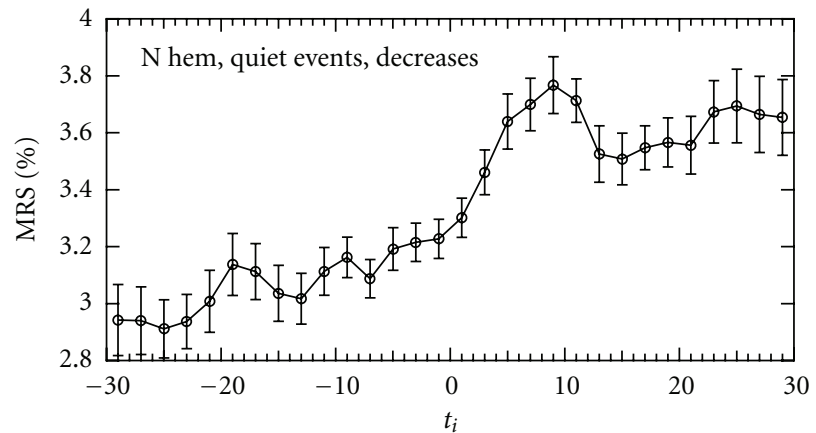

(c)

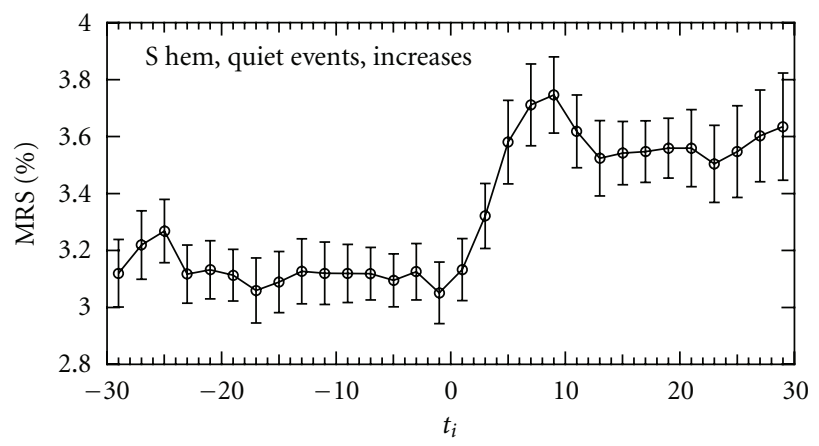

(b)

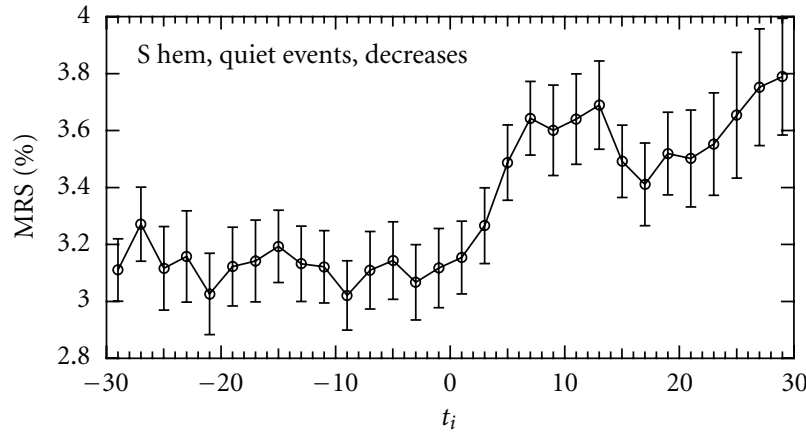

(d)

FIgURE 1: MRS as a function of the scan time, $t_{i}$, for Quiet events: (a) NH Is; (b) SH Is; (c) NH Ds; (d) SH Ds.

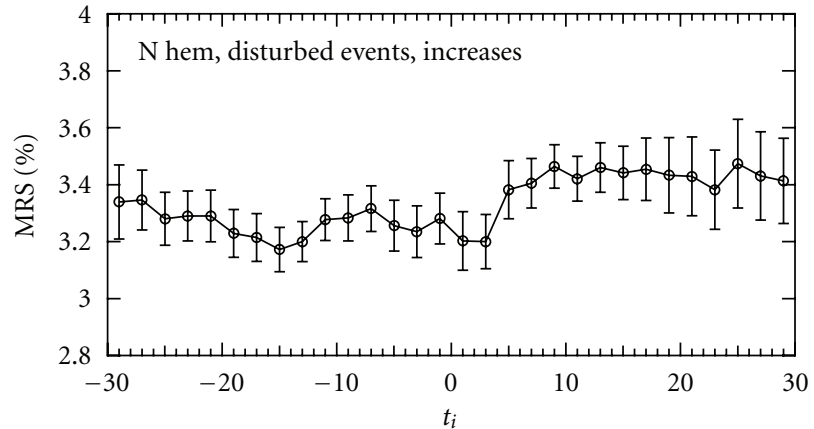

(a)

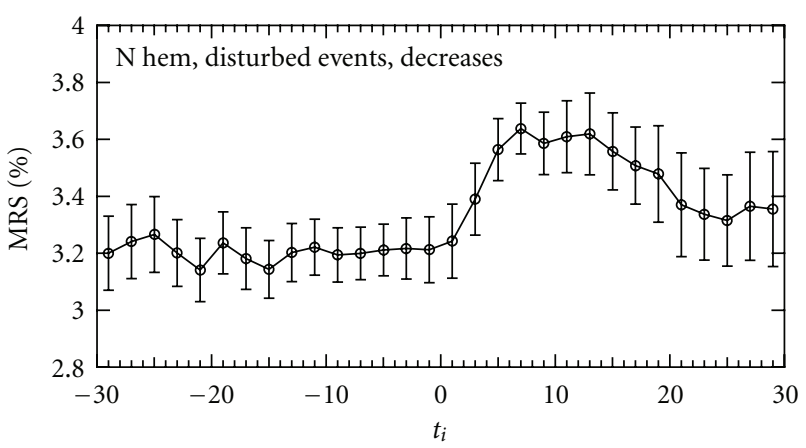

(c)

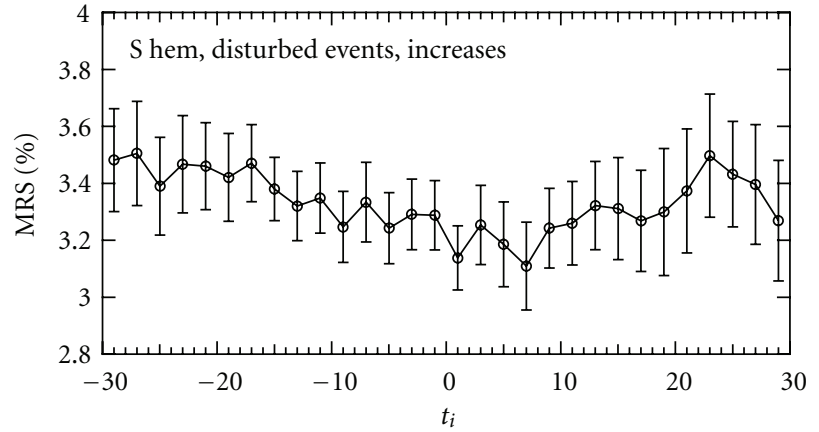

(b)

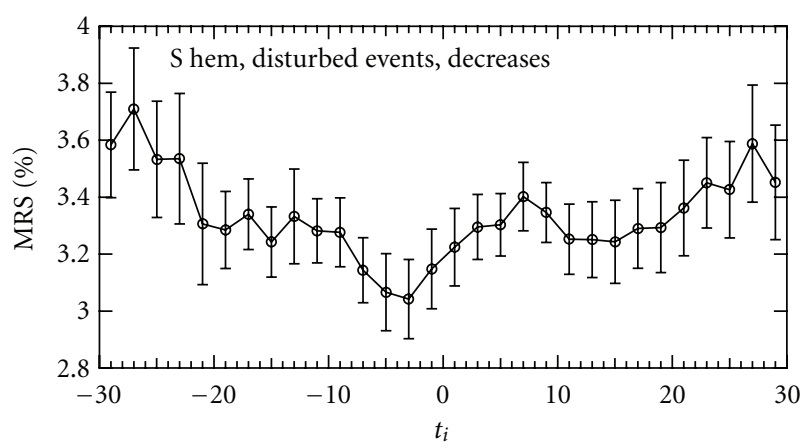

(d)

FIGURE 2: MRS as a function of the scan time, $t_{i}$, for Disturbed events: (a) NH Is; (b) SH Is; (c) NH Ds; (d) SH Ds. 
TABLE 2: An overview of the echoes statistics for the different subsets of the events used for the PCP analysis. Is and Ds stand for Increases and Decreases of solar wind dynamic pressure, $\mathrm{NH}$ and $\mathrm{SH}$ stand for Northern and Southern Hemisphere.

\begin{tabular}{lcc}
\hline Set of PCP events & Number of events & Number of echoes \\
\hline Quiet Is NH & 31 & 1077530 \\
Quiet Is SH & 21 & 238995 \\
Quiet Ds NH & 14 & 610360 \\
Quiet Ds SH & 13 & 362373 \\
Disturbed Is NH & 21 & 683244 \\
Disturbed Is SH & 21 & 421303 \\
Disturbed Ds NH & 13 & 608818 \\
Disturbed Ds SH & 15 & 339407 \\
\hline
\end{tabular}

the measured vectors is the minimum required to constrain the spherical harmonic expansion at every order and in all the points of the space grid. The spatial distribution of the measured velocities is a feature even more important than the mere number of data points: the symmetries of the ionospheric convection are such that few points located in strategic regions (e.g., throats or points of flux reversal of the convection cells) often allow a better reconstruction of the whole potential pattern than a denser distribution of points concentrated in the core of a single convection cell only.

In order to test the weight of the model data with respect to the measured data, for each event of our data base, 25 convection maps were generated: one using "true" IMF data from ACE (delayed by $T_{g}$ ) and the others obtained imposing fixed values of IMF, in order to fall in all the different cases of the RG96 convection model. For each scan $i$ of each event $j$ average values $\mu_{k}^{j}(i)$ and standard deviations $\sigma_{k}^{j}(i)$ were calculated over the set of the $25 \mathrm{CPCP}\left(\Delta V_{k}\right)$ calculated from the maps. Let $N_{j}(i)$ be the number of $\Delta V_{k}$ so that $\Delta V_{k}>$ $\mu_{k}^{j}(i)+\sigma_{k}^{j}(i)$ or $\Delta V_{k}<\mu_{k}^{j}(i)-\sigma_{k}^{j}(i)$. If $N_{j}(i) \leq 8$ for at least the $70 \%$ of the scans, $i$, the event $j$ has been taken as good. This just means that the velocity distribution is good enough so that the calculated CPCP is reliable: data dominate over the model.

Given the new set of events, $h$, survived from the above criterion, for each scan, $i$, up to 5 CPCP over 25 are removed (the 3 lowest and the 2 highest values), and new averages $\mu_{h i}^{\prime}$ and errors $\epsilon_{h i}=\sigma_{h i}^{\prime} / \sqrt{20}$ have been calculated. For a given set of $N$ events, one can finally calculate the average CPCP for each scan, $V_{\mathrm{av}}(i)$ as: $V_{\mathrm{av}}(i)=$ $\left[\sum_{j=1, N} \mu_{j}^{\prime}(i)\right] / N$. The associated errors are obtained using the standard propagation formula [19].

3.1. Results of the CPCP Statistics. Table 2 summarizes the characteristics of the data base after the severe selection imposed by the criteria described above. The number of events and echoes has been dramatically reduced for all the subsets, so that one cannot completely exclude that one or few particular cases influence the statistics. Moreover the distribution of echoes and events is no more homogeneous all over the years (not shown in the table), and seasonal effects can occur as well. However, in the subsets where a clear trend can be recognized, careful inspections of the single events confirmed the overall trends.

Figure 3 shows the average CPCP $V_{\mathrm{av}}(i)$ as a function of the scan time, $t_{i}$ for Quiet events: Figure 3(a) is for $\mathrm{NH}$ Is, Figure 3(b) is for SH Is, Figure 3(c) is for NH Ds, and Figure $3(\mathrm{~d})$ is for $\mathrm{SH}$ Ds. The clearest behaviours show up for the NH Is, where an average increase of $2.5 \mathrm{kV}$ of CPCP is observed very well correlated with the SIs occurrence, and for the SH Ds, where the CPCP starts to increase shortly after $T_{g}$, but more gradually.

Figure 4 shows the same quantity for Disturbed events with the same arrangements as for Figure 3. No clear trends can be inferred from the data: the CPCP is highly variable and there are not particular changes in the curves that could be associated to the SIs occurred at $T_{g}$.

\section{Discussion and Conclusions}

The results for Quiet Is seem to confirm the previous results by Co2005, also for the SH. We propose the following interpretation: when the pressure pulse hits the magnetopause, a fast compressional MHD wave is launched, and couples to a shear Alfvén wave at a resonance position which depends on the local Alfvén speed (e.g., Samson et al. [20]); this results in a field line resonance which induces soft particle precipitation in the $\mathrm{F}$ region and a consequent increase of the ionospheric irregularities and of MRS. On the other hand, the precipitation of trapped electrons due to loss cone instability induced by the SIs in the magnetosphere can also account for the MRS increase.

An interesting new result which shows up from the extended data base is the average increase of the echoes in both Hemispheres for the Quiet Ds set. This seems to confirm the hypothesis by Araki [2] that a sudden decrease of the solar wind dynamic pressure should give rise to the same kind of current systems in the magnetosphere and the ionosphere as for a sudden increase: ground-based high-latitude magnetometers usually show the signatures of pairs of vortex-like current structures in the ionosphere, induced by Alfvén waves aligned with the geomagnetic field, and symmetric across noon. The direction of the currents (clockwise or counterclockwise) depends on local time and on the nature of the pressure pulse. Very few studies exist for decreases of the solar wind pressure: Araki and Nagano [21] showed that the Araki model is consistent for both increases and decreases of the pressure, while Takeuchi et al. $[22,23]$ found that Ds provoke more or less the same currents system as Is, and there are no appreciable differences in the distribution and current circulation of the vortices pairs. Nevertheless, an energy transfer from the pressure discontinuity to the ionosphere occurs all the same, capable of exciting field-aligned waves and current vortices: as a consequence, an average increase of the ionospheric irregularities is observed.

As for the Disturbed events sets, the new results show no evidence of the decrease of the radar echoes reported by Co2005: during high active periods, the ionosphere often 


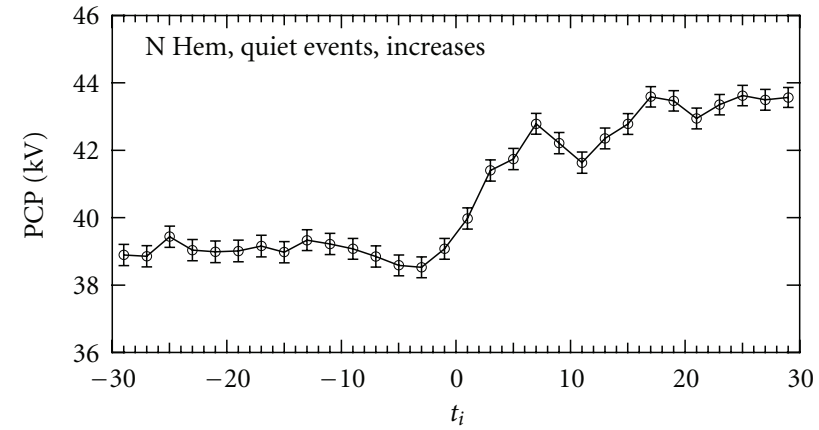

(a)

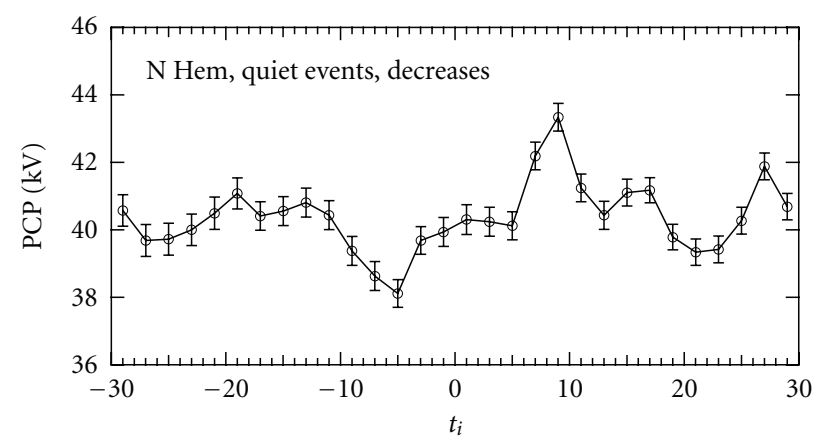

(c)

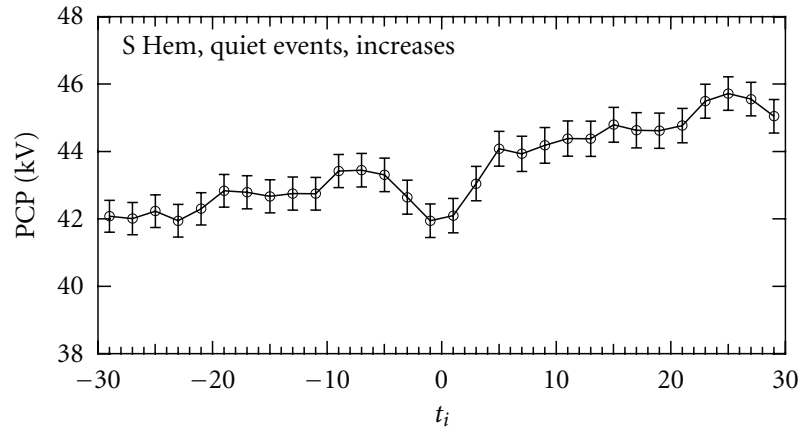

(b)

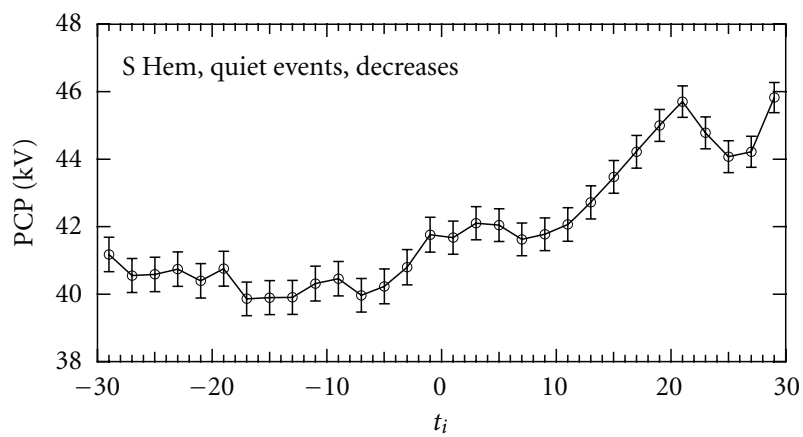

(d)

FIGURe 3: $V_{\mathrm{av}}(i)$ (PCP) as a function of the scan time, $t_{i}$, for Quiet events: (a) NH Is; (b) SH Is; (c) NH Ds; (d) SH Ds.

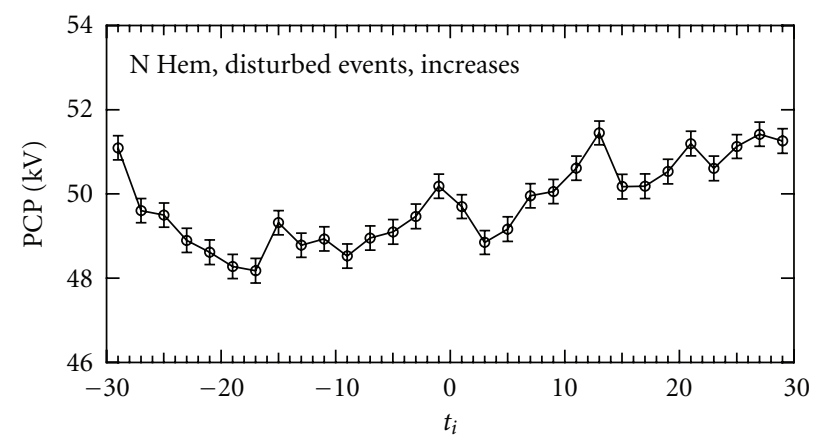

(a)

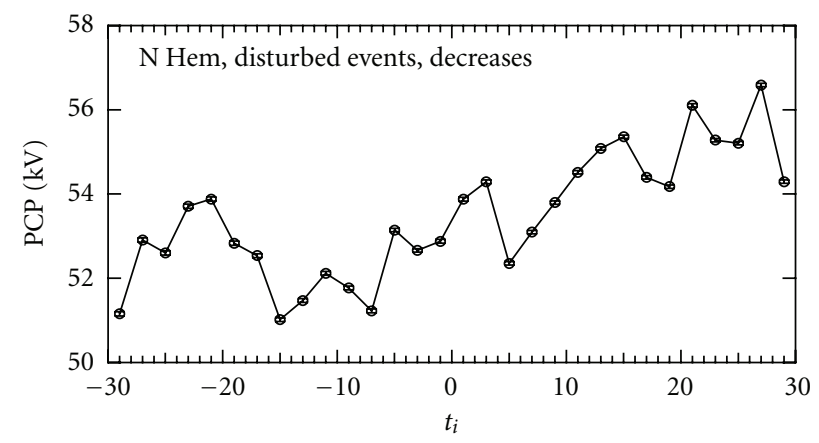

(c)

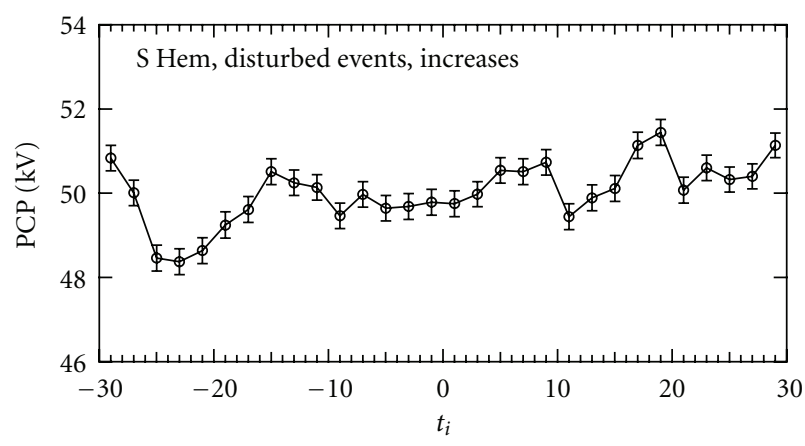

(b)

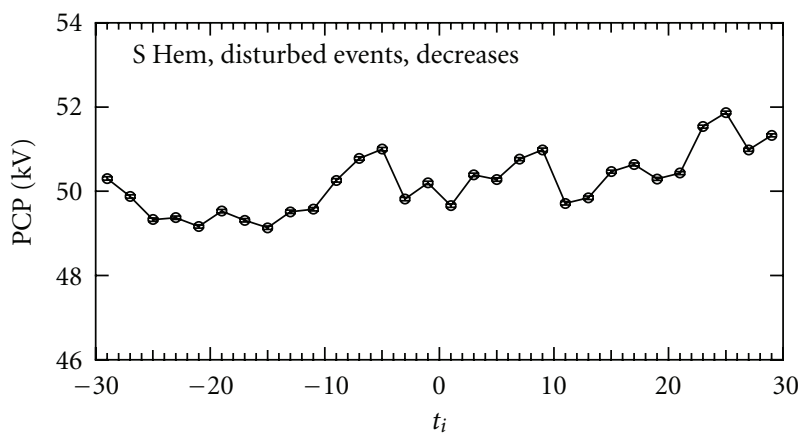

(d)

FIgURE 4: $V_{\mathrm{av}}(i)(\mathrm{PCP})$ as a function of the scan time, $t_{i}$, for Disturbed events: (a) NH Is; (b) SH Is; (c) NH Ds; (d) SH Ds. 
suffers the effects of geomagnetic storms and substorms, so that the occurrence of an SI can be masked by other phenomena already at play. Where some particular behaviour can be isolated, there is rather an increase of the echoes $(\mathrm{NH}$ Is and Ds). It has also to be noted that a selection of the events according to the IMF ( $B_{z}$ or the clock angle) has not yet been done for the new database; Co2005, for example, found that all the behaviours of the echoes were stronger when IMF $B_{z}$ was negative, as one would expect, because of the greater amount of energy transferred from solar wind to the magnetosphere and the ionosphere with such IMF orientation. Furthermore, a future study will possibly join the data bases of Co2005 and that of the present work together, in order to encompass at least one complete solar cycle and allow to test the echo response as a function of solar activity and seasonal variations.

Unfortunately the statistics dramatically diminish when one attempts to take into account the CPCP, because a reliable evaluation of this quantity requires very good data coverage. Nevertheless, encouraging preliminary results have been obtained which confirm the increase of CPCP caused by a pressure pulse for Quiet events. Again the effect of an increased energy transfer from the solar wind to the magnetosphere and the ionosphere during SIs, is possibly masked during disturbed periods.

\section{Acknowledgments}

The authors would like to thank N. Ness and D. McComas for the use of ACE Magnetic Field Instrument and Solar Wind Experiment data, respectively; the CDAWeb team; the World Data Center for Geomagnetism, Kyoto, Japan, for providing $\mathrm{AE}$ index and equatorial magnetograms; the principal investigators of the SuperDARN radars in the Northern and Southern Hemispheres. This work is supported by the Italian National Program for the Antarctic Research (PNRA) under projects 2004/7.1 and 2004/7.2; I. Coco thanks the Scientific Committee for Antarctic Research (SCAR) for the financial support through the SCAR Fellowship Program 2007-2008. This work was partially supported by ASI under Contract no. I/015/07/0 "Studi di Esplorazione del Sistema Solare".

\section{References}

[1] D. G. Sibeck, "A model for the transient magnetospheric response to sudden solar wind dynamic pressure variations," Journal of Geophysical Research, vol. 95, pp. 3755-3771, 1990.

[2] T. Araki, "A physical model of the geomagnetic sudden commencement, in "solar wind sources of magnetospheric ultra-low-frequency waves"'” Geophysical Monograph Series, vol. 81, no. 270, pp. 183-200, 1994.

[3] A. Boudouridis, E. Zesta, L. R. Lyons, P. C. Anderson, and D. Lummerzheim, "Effect of solar wind pressure pulses on the size and strength of the auroral oval," Journal of Geophysical Research, vol. 108, no. A4, 2003.

[4] A. Boudouridis, E. Zesta, L. R. Lyons, P. C. Anderson, and D. Lummerzheim, "Enhanced solar wind geoeffectiveness after a sudden increase in dynamic pressure during southward IMF orientation," Journal of Geophysical Research, vol. 110, no. 5, 2005.

[5] G. Chisham, M. P. Freeman, G. A. Abel et al., "Remote sensing of the spatial and temporal structure of magnetopause and magnetotail reconnection from the ionosphere," Reviews of Geophysics, vol. 46, no. 1, 2008.

[6] G. Provan, M. Lester, S. W. H. Cowley et al., "Modulation of dayside reconnection during northward interplanetary magnetic field," Journal of Geophysical Research, vol. 110, no. A10, 2005.

[7] I. Coco, E. Amata, M. F. Marcucci, D. Ambrosino, J. P. Villain, and C. Hanuise, "The effects of an interplanetary shock on the high-latitude ionospheric convection during an IMF $\mathrm{B}_{y}$ -dominated period," Annales Geophysicae, vol. 26, no. 9, pp. 2937-2951, 2008.

[8] R. A. Greenwald, K. B. Baker, J. R. Dudeney et al., "DARN/SuperDARN - a global view of the dynamics of highlatitude convection," Space Science Reviews, vol. 71, no. 1-4, pp. 761-796, 1995.

[9] G. Chisham, M. Lester, S. E. Milan et al., "A decade of the Super Dual Auroral Radar Network (SuperDARN): scientific achievements, new techniques and future directions," Surveys in Geophysics, vol. 28, no. 1, pp. 33-109, 2007.

[10] X. Zhou and B. T. Tsurutani, "Rapid intensification and propagation of the dayside aurora: large scale interplanetary pressure pulses (fast shocks)," Geophysical Research Letters, vol. 26, no. 8, pp. 1097-1100, 1999.

[11] J. P. Villain, C. Beghin, and C. Hanuise, "ARCAD3-SAFARI coordinated study of auroral and polar F-region ionospheric irregularities," Annales Geophysicae, vol. 4, pp. 61-68, 1986.

[12] P. Ballatore, J. P. Villain, N. Vilmer, and M. Pick, "The influence of the interplanetary medium on SuperDARN radar scattering occurrence," Annales Geophysicae, vol. 18, pp. 15761583, 2001.

[13] J. A. Wild and A. Grocott, "The influence of magnetospheric substorms on SuperDARN radar backscatter," Journal of Geophysical Research, vol. 113, no. 4, 2008.

[14] I. Coco, E. Amata, M. F. Marcucci et al., "Effects on SuperDARN HF radar echoes of sudden impulses of solar wind dynamic pressure," Annales Geophysicae, vol. 23, no. 5, pp. 1771-1783, 2005.

[15] T. N. Davis and M. Sugiura, "Auroral electrojet activity index $\mathrm{AE}$ and its universal time variations," Journal of Geophysical Research, vol. 71, p. 785, 1966.

[16] J. M. Ruohoniemi and K. B. Baker, "Large-scale imaging of high-latitude convection with Super Dual Auroral Radar Network HF radar observations," Journal of Geophysical Research, vol. 103, no. 9, pp. 20797-20811, 1998.

[17] J. M. Ruohoniemi and R. A. Greenwald, "Statistical patterns of high-latitude convection obtained from Goose Bay HF radar observations," Journal of Geophysical Research, vol. 101, pp. 21 743-21 763, 1996.

[18] S. G. Shepherd and J. M. Ruohoniemi, "Electrostatic potential patterns in the high-latitude ionosphere constrained by SuperDARN measurements," Journal of Geophysical Research, vol. 105, no. 10, pp. 23005-23014, 2000.

[19] J. H. Taylor, An Introduction to Error Analysis, The Study of Uncertainties in Physical Measurements, chapter 3, University Science Books, 1982.

[20] J. C. Samson, R. Rankin, and V. T. Tikhonchuk, "Optical signatures of auroral arcs produced by field line resonances: comparison with satellite observations and modeling," Annales Geophysicae, vol. 21, no. 4, pp. 933-945, 2003. 
[21] T. Araki and H. Nagano, "Geomagnetic response to sudden expansions of the magne tosphere," Journal of Geophysical Researc, vol. 93, p. 3983, 1988.

[22] T. Takeuchi, T. Araki, H. Luehr et al., "Geomagnetic negative sudden impulse due to a magnetic cloud observed on May 13, 1995," Journal of Geophysical Research, vol. 105, no. 8, pp. 18835-18846, 2000.

[23] T. Takeuchi, T. Araki, A. Viljanen, and J. Watermann, "Geomagnetic negative sudden impulses: interplanetary causes and polarization distribution," Journal of Geophysical Research, vol. 107, no. 7, p. 1096, 2002. 

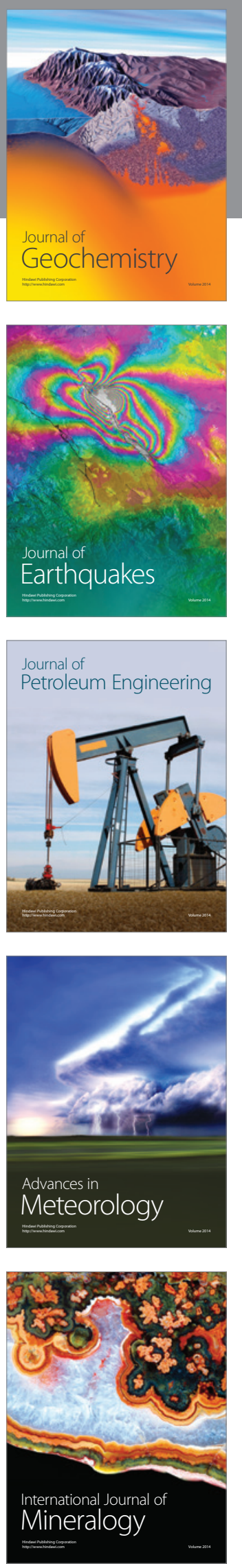
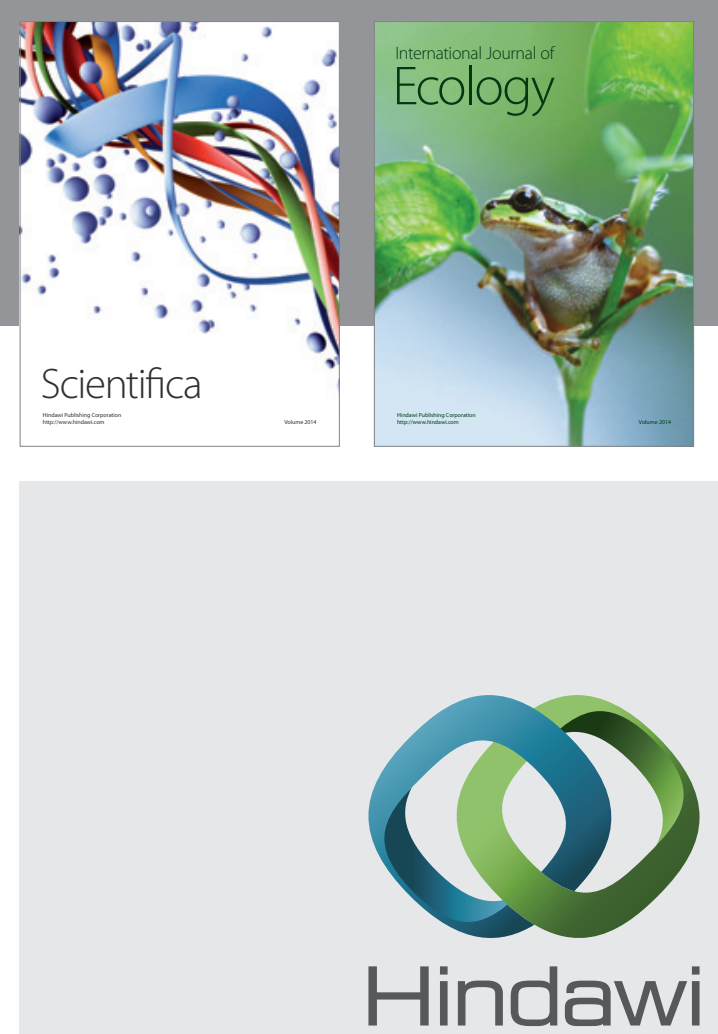

Submit your manuscripts at http://www.hindawi.com
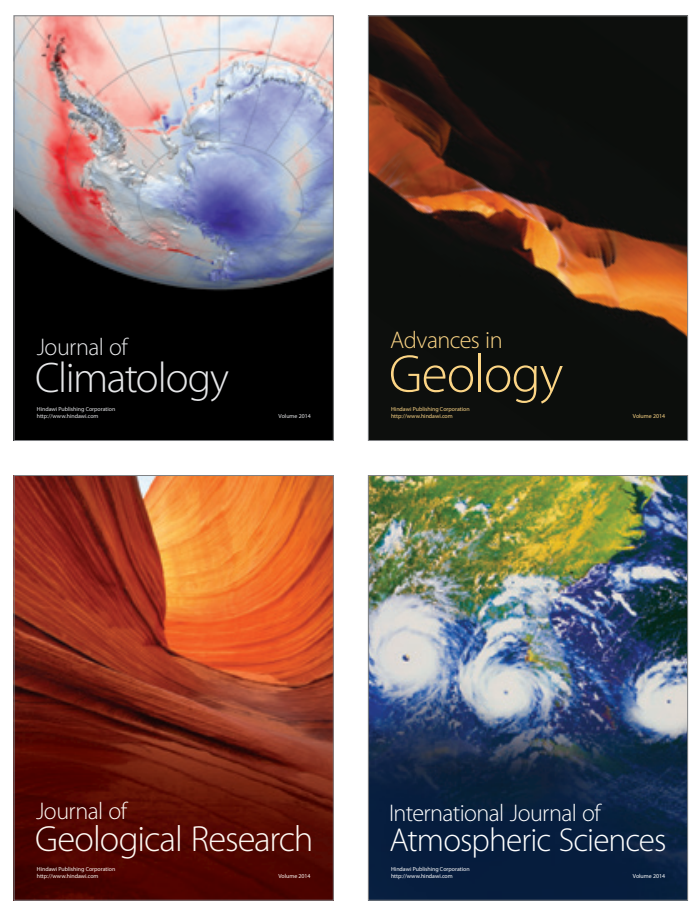
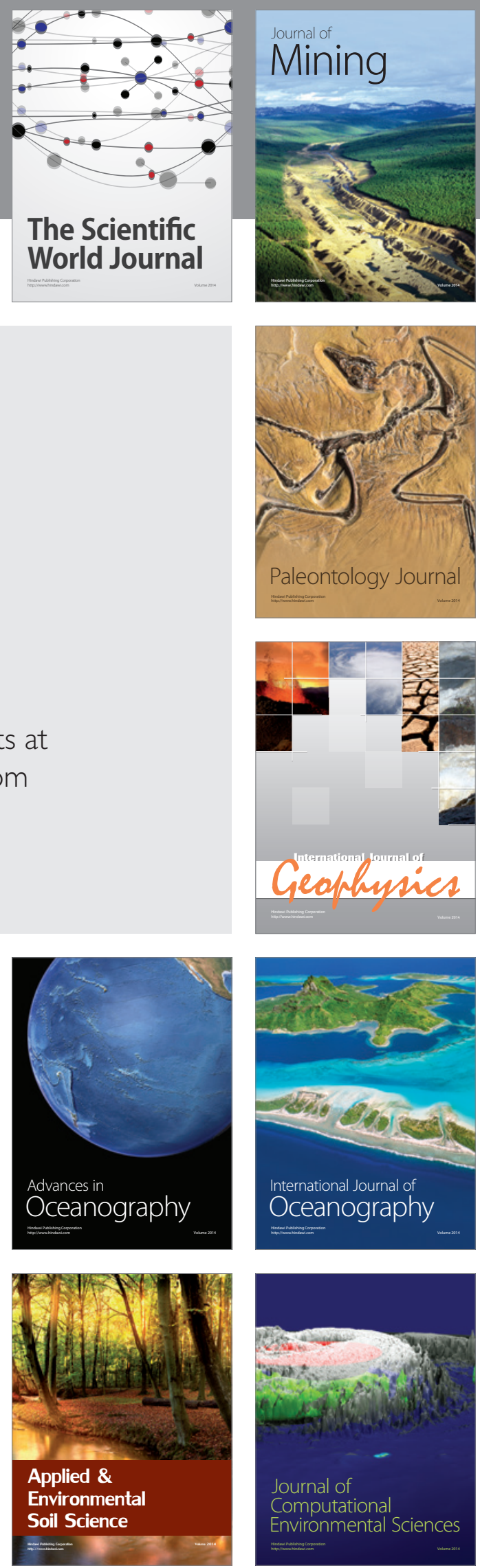